weight, height, ethnicity) and spirometry nearest to 6 years of age because repeatable measurements in a clinical context are feasible at this age. The primary outcome was $\mathrm{FEV}_{1} \%$ predicted (\%p) (GLI reference equation (http://www.lungfunction.org/); and VS at annual assessment. Between 1-5 scans were performed prior to the age 6 year spirometry, and were independently reported as normal or abnormal (at least one abnormal VS). Statistical analysis was performed using Student t test. $\mathrm{P}<0.05$ was considered significant.

Results 143/217 children (72 females, 71 males) had data on VS and spirometry available; mean age at first spirometry was 6.36 (range 5.0-7.6). The remaining 73 were excluded due to late diagnosis, moving away before the first reliable spirometry, or first being seen later than the window for ventilation scans $(1-5$ years). Children with $\geq 1$ abnormal VS had a statistically significant reduction in lung function (mean FEV1\% p 83.4\%) when compared with children with normal ventilation scans (mean FEV1\% p\%89.6), $\mathrm{P}=0.03$ (Figure 1).

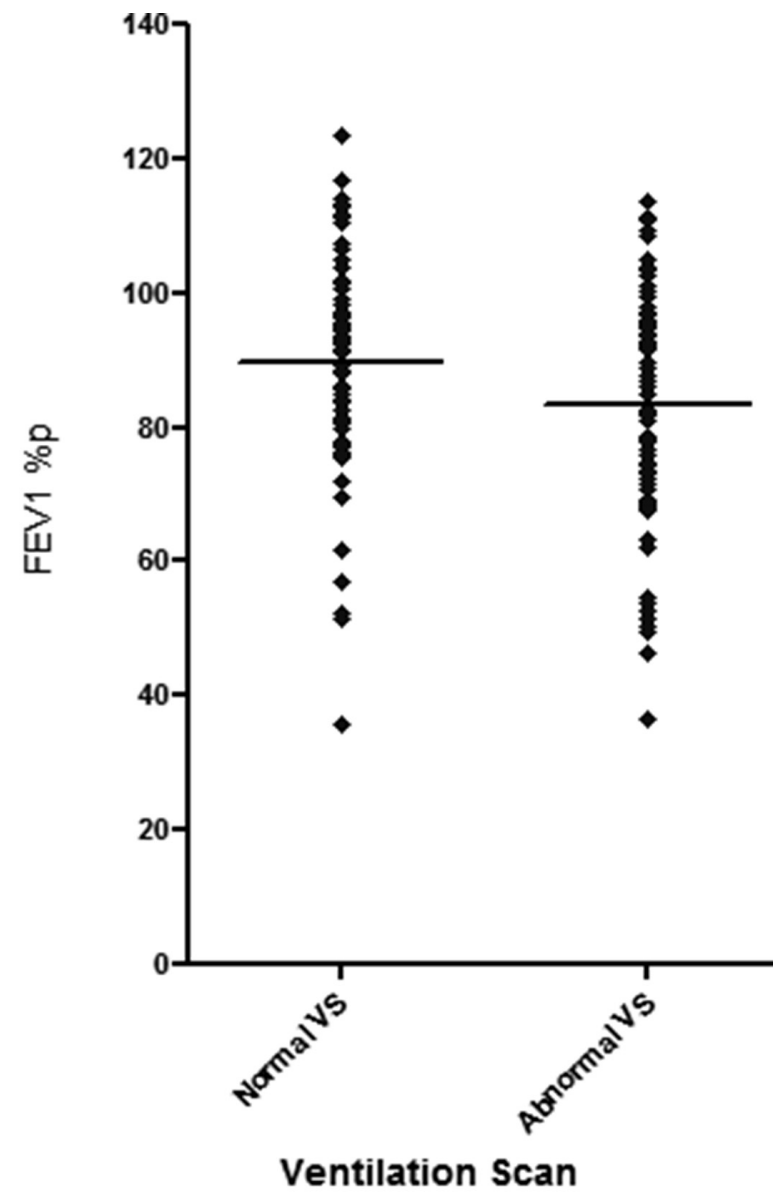

Abstract S17 Figure 1 Dot plot showing\%p comparison between those with normal and abnormal scans. The black horizontal lines are the group means

Conclusion Although abnormal VS predict abnormal first spirometry, the overlap between the two groups means that VS are not a useful clinical tool to delineate a high risk group.

\section{S18 IVACAFTOR TREATMENT IN PRESCHOOL CHILDREN WITH CYSTIC FIBROSIS AND A CFTR GATING MUTATION: EXTENDED EVALUATION}

${ }^{1} \mathrm{JC}$ Davies, ${ }^{2} \mathrm{~S}$ Cunningham, ${ }^{3} \mathrm{KW}$ Southern, ${ }^{4} \mathrm{~S}$ Robertson, ${ }^{5} \mathrm{Y}$ Green, ${ }^{5} \mathrm{~J}$ Cooke, ${ }^{5} \mathrm{M}$ Higgins, ${ }^{6} \mathrm{M}$ Rosenfeld. ${ }^{1}$ Imperial College \& Royal Brompton \& Harefield NHS Foundation Trust, London, UK; ${ }^{2}$ Royal Hospital for Sick Children, Edinburgh, UK; ${ }^{3}$ Alder Hey Children's Hospital, Liverpool, UK; ${ }^{4}$ Vertex Pharmaceuticals Incorporated, Boston, USA; ${ }^{5}$ Vertex Pharmaceuticals (Europe) Limited, Milton Park, UK; ${ }^{6}$ Seattle Children's Hospital, Seattle, USA

\subsection{6/thoraxjn-2015-207770.24}

Introduction and objectives Ivacaftor, a cystic fibrosis transmembrane conductance regulator (CFTR) potentiator, has demonstrated improved outcomes in patients aged $\geq 6$ years with cystic fibrosis (CF) and a CFTR gating mutation. The phase 3, openlabel, 24-week KIWI study assessed safety and pharmacokinetics of ivacaftor (50- or $75-\mathrm{mg}$ granules twice daily [bid] for weight. Methods Patients who completed KIWI could enrol in KLIMB (an 84-week, open-label extension study). Dosing was the same as in KIWI for patients aged $<6$ years; for patients aged $\geq 6$ years, dosing was 150-mg tablets bid.

Results KIWI enrolled 34 patients (mean age, 3.2 years); 33 enrolled in KLIMB. Cough was the most common AE in both KIWI (56\%) and KLIMB (64\%). Over the total 72-week treatment period, 8 patients had ALT or AST elevations of $>8 \times$ the upper limit of normal (ULN), 6 of whom had liver function tests (LFTs) $>2 \times$ ULN at pretreatment baseline. Six of the 8 patients with LFTs $>8 \times$ ULN had drug interruption; study drug was subsequently resumed. In total, 3 patients permanently discontinued study drug (elevated LFTs, $\mathrm{n}=2$; needle phobia, $\mathrm{n}=1$ ). Improvements in sweat chloride, faecal elastase-1, and immunoreactive trypsinogen were maintained over 72 weeks. Overall improvements from baseline in other exploratory outcome measures were also observed (Table 1).

Conclusions A favourable overall safety profile was demonstrated with ivacaftor during extended follow-up in preschool patients with CF. Reported adverse events were consistent with the known safety profile of ivacaftor; additional monitoring of liver function may be required in this age group, particularly in patients with a history of elevated LFTs. Improvements in markers of pancreatic function and sweat chloride were sustained over the extended follow-up.

\begin{tabular}{lll}
$\begin{array}{l}\text { Abstract S18 Table 1 } \\
\text { measures }\end{array}$ & Summary of exploratory efficacy outcome \\
\hline $\begin{array}{l}\text { Absolute change from KIWI } \\
\text { baseline, mean (SD) }\end{array}$ & KIWI (Wk 24) & KLIMB (Wk 72) \\
\hline Sweat chloride, mmol/L & $-46.9(26.2) P<0.0001$ & $-45.4(31.3) P<0.0001$ \\
Weight, kg & $1.4(0.6) P<0.0001$ & $3.2(1.1) P<0.0001$ \\
Weight-for-age z-score & $0.2(0.3) P<0.0001$ & $0.1(0.5) P=0.3$ \\
BMI, kg/m ${ }^{2}$ & $0.3(0.5) P=0.002$ & $-0.2(0.7) P=0.2$ \\
BMI-for-age z-score & $0.4(0.4) P<0.0001$ & $0.1(0.5)=0.5$ \\
Stature, cm & $3.3(1.2) P<0.0001$ & $10.2(1.8) P<0.0001$ \\
Stature-for-age z-score & $-0.01(0.3) P=0.8$ & $0.1(0.3) P=0.01$ \\
Fecal elastase-1, $\mu \mathrm{gg} / \mathrm{g}^{\mathrm{a}}$ & $99.8(138.4)$ & $101.9(152.3)$ \\
Immunoreactive trypsinogen, $\mathrm{ng} / \mathrm{mL}^{\mathrm{a}}$ & $-20.7(24.0)$ & $-18.9(28.0)$
\end{tabular}

$P$ values for both KIWI and KLIMB assessed for absolute change from KIWI baseline. ${ }^{a}$ Significance was not assessed for these changes. 


\section{TIME to change: management of pleural disease}

\section{S19 INTERVENTIONS FOR THE MANAGEMENT OF MALIGNANT PLEURAL EFFUSIONS}

${ }^{1} \mathrm{AO}$ Clive, ${ }^{1} \mathrm{HE}$ Jones, ${ }^{1} \mathrm{R}$ Bhatnagar, ${ }^{2} \mathrm{NJ}$ Preston, ${ }^{1} \mathrm{NA}$ Maskell. ${ }^{1}$ University of Bristol, Bristol, $U K_{;}{ }^{2}$ Lancaster University, Lancaster, UK

10.1136/thoraxjnl-2015-207770.25

Aims Malignant pleural effusion (MPE) is a common clinical problem and a number of treatment options are available to manage these patients.

We undertook a systematic review of the literature and metaanalysis in order to ascertain the optimal management strategy for adults with symptomatic MPE.

Methods We searched CENTRAL, MEDLINE, EMBASE, CINAHL; SCI-EXPANDED and SSCI (ISI Web of Science) databases to May 2015. We included randomised controlled trials of intrapleural interventions for adults with symptomatic MPE. Two review authors independently extracted the data and assessed the studies' risk of bias.

The primary outcome measure was pleurodesis failure rate. Secondary outcome measures were adverse effects and complications, patient reported control of breathlessness, quality of life, cost, mortality, duration of inpatient stay and patient acceptability.

We performed network meta-analysis with random effects to analyse the primary outcome data and those secondary outcomes with enough data. If this was not possible, we reported the results by narrative synthesis.

Results Of the 1888 records identified, 62 randomised trials, including a total of 3428 patients, were eligible for inclusion. All studies were at high risk of bias for at least one domain and the majority were unblinded.

Network meta-analysis evaluating the rate of pleurodesis failure suggested Talc Poudrage to be the most effective method (estimated rank 1 [95\% CI 1, 4]). The estimated ranks of the other evaluated methods are shown in the Figure. The estimates were imprecise as evidenced by the wide credible intervals. Both statistical and clinical heterogeneity was high.

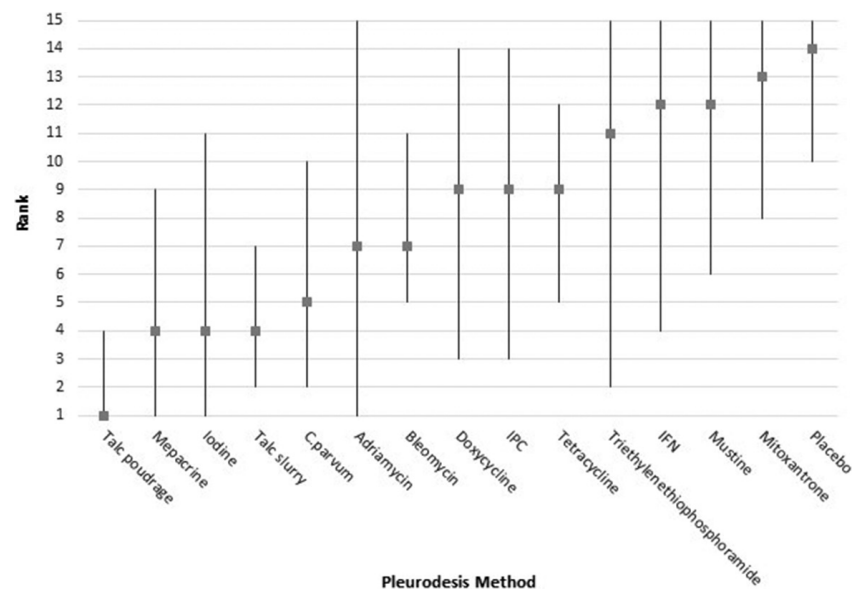

Abstract S19 Figure 1 Estimated ranks (95\% Cr-l) for each of the pleurodesis methods from the main network meta-analysis

The secondary outcomes were inconsistently reported. Network meta-analysis was only performed for pain, fever and mortality and minimal evidence was obtained suggesting differences between treatments for these outcomes. Indwelling pleural catheters were examined in two RCTs, both reporting improved breathlessness when compared to Talc Slurry pleurodesis, despite lower pleurodesis success rates.

Conclusions Based on the available evidence, Talc Poudrage may be the optimal method for obtaining a pleurodesis in MPE. However, there is minimal evidence to suggest large differences between the next most effective methods. Global experience of these agents and their adverse events must also be considered when selecting a sclerosant.

\section{S20 PRIMARY RESULT OF THE 1ST THERAPEUTIC INTERVENTIONS IN MALIGNANT EFFUSION (TIME1) TRIAL: A 2 × 2 FACTORIAL, RANDOMISED TRIAL OF CHEST TUBE SIZE AND ANALGESIC STRATEGY FOR PLEURODESIS IN MALIGNANT PLEURAL EFFUSION}

${ }^{1} \mathrm{NM}$ Rahman, ${ }^{2} \mathrm{~J}$ Pepperell, ${ }^{3} \mathrm{~S}$ Rehal, ${ }^{4} \mathrm{~T}$ Saba, ${ }^{4} \mathrm{~A}$ Tang, ${ }^{5} \mathrm{~N}$ Ali, ${ }^{6} \mathrm{~A}$ West, ${ }^{6} \mathrm{G}$ Hettiarachchi, ${ }^{7} \mathrm{D}$ Mukherjee, ${ }^{7} \mathrm{~J}$ Samuel, ${ }^{8} \mathrm{~A}$ Bentley, ${ }^{9} \mathrm{~L}$ Dowson, ${ }^{10} \mathrm{~J}$ Miles, ${ }^{11} \mathrm{~F}$ Ryan, ${ }^{12} \mathrm{~K}$ Yoneda, ${ }^{13} \mathrm{~A}$ Chauhan, ${ }^{1} \mathrm{~J}$ Corcoran, ${ }^{1} \mathrm{I}$ Psallidas, ${ }^{1} \mathrm{JM}$ Wrightson, ${ }^{1} \mathrm{R}$ Hallifax, ${ }^{14} \mathrm{HE}$ Davies, ${ }^{15} Y$ CG Lee, ${ }^{1}$ EL Hedley, ${ }^{16} \mathrm{D}$ Seaton, ${ }^{1} \mathrm{~N}$ Russell, ${ }^{1} \mathrm{M}$ Chapman, ${ }^{1} \mathrm{BM}$ McFadyen, ${ }^{1} \mathrm{RA}$ Shaw, ${ }^{1} \mathrm{RJO}$ Davies, ${ }^{17} \mathrm{NA}$ Maskell, ${ }^{3} \mathrm{AJ}$ Nunn, ${ }^{18} \mathrm{RF}$ Miller. ${ }^{1}$ Oxford Respiratory Trials Unit, Oxford, UK; ${ }^{2}$ Somerset Lung Centre, Musgrove Park Hospital, Taunton, UK; ${ }^{3}$ Medical Research Council Clinical Trials Unit, University College London, London, UK; ${ }^{4}$ Blackpool, Fylde and Wyre Hospitals NHS Trust, Blackpool, UK: ${ }^{5}$ King's Mill Hospital, Mansfield, UK: ${ }^{6}$ Medway Maritime Hospital, Gillingham, UK; ${ }^{7}$ Basildon University Hospital, Basildon, UK; ${ }^{8}$ University Hospital South Manchester NHS Trust, Manchester, UK; ${ }^{9}$ Royal Wolverhampton Hospital NHS Trust, Wolverhampton, UK; ${ }^{10}$ Rotherham General Hospital, Rotherham, UK; ${ }^{11}$ Vancouver Coastal Health, Vancouver, Canada; ${ }^{12}$ UC Davis Medical Centre, Sacramento, USA; ${ }^{13}$ Queen Alexandra Hospital, Portsmouth, UK; ${ }^{14}$ Cardiff and Vale University Health Board, Cardiff, UK; ${ }^{15}$ School of Medicine \& CAARR, University of Western Australia, Perth, Australia; ${ }^{16}$ Department of Respiratory Medicine, Ipswich Hospital, Ipswich, UK; ${ }^{17}$ Academic Respiratory Unit, Department of Clinical Sciences, Southmead Hospital, University of Bristol, Bristol, UK; ${ }^{18}$ Research Department of Infection and Population Health, Institute of Epidemiology and Healthcare, University College London, London, UK

\subsection{6/thoraxjnl-2015-207770.26}

Background Optimal management of pleurodesis for malignant pleural effusion (MPE) has not been defined either in terms of optimal analgesia or chest tube size. Non-steroidal anti-inflammatory drugs (NSAID) are highly effective analgesics, but are avoided in pleurodesis as they may reduce pleurodesis efficacy. Smaller $(<14$ French) chest tubes may be less painful compared to larger chest tubes, but their efficacy in MPE pleurodesis has not been proven. This study investigated chest tube size (large versus small) and analgesia (NSAID versus opiate) in this setting. Methods A $2 \times 2$ factorial, phase 3 randomised controlled trial in 320 patients with MPE undergoing pleurodesis. Patients were randomised to opiate/NSAID and 24 French drain/12 French drain. Co-primary outcomes were; pain while tube in situ, measured on $100 \mathrm{~mm}$ visual analogue scale (VAS) over 5 days (superiority comparison) and pleurodesis efficacy at 3 months (noninferiority comparison, margin of non-inferiority 15\%). Secondary outcomes included use of rescue analgesia, pleurodesis success to 6 months, adverse events and mortality.

Results 320 patients were randomised (63\% male, mean age 71.8 years), with similar baseline characteristics. Mean VAS scores in opiate and NSAID groups were similar (adjusted mean difference, $-1.5 \mathrm{~mm}$ (95\% confidence interval [CI], -5.0 to 2.0; $\mathrm{p}=0.40)$. Patients receiving NSAID required more rescue analgesia (38\% vs. 26\%). Pleurodesis failure occurred in 33/144 (23\%) NSAID patients compared with 30/150 (20\%) of participants receiving opiate, meeting criteria $(15 \%)$ for non-inferiority (difference 3\%; $(90 \%$ CI $-5 \%$ to $10 \%)$ ). Smaller chest tubes 